\title{
Cultural Translation in English Web Pages of Shaanxi Universities from the Perspective of Eco-Translatology
}

\author{
Chunyan Jing \\ School of Humanities \& Social Sciences, Xi'an Polytechnic University, Shaanxi, Xi'an 710048, \\ China \\ chunyanjing@yeah.net
}

Keywords: Ecological translation; English web page; Cultural translation

\begin{abstract}
The English cultural characteristic words carry the unique culture of English, reflecting the history, geography, customs, traditional customs, lifestyle, literature and art, behavioral norms, ways of thinking, values, etc. of the English-speaking countries, with high social and cultural values. And linguistic value. The Chinese translation of English cultural characteristic words can be translated from the perspective of ecological translation studies using the translation standards and principles of ecological translation studies. Ecological translation studies focus on overall/association, stressing dynamics/balance, embodying ecological aesthetics, caring for "translation community", indicating translation ethics and advocating diverse/unified ecological rationality. This paper intends to use language translation, language dimension, cultural dimension, Analyze the corpus at three levels of communication, analyze the differences in the ecological effects of Chinese-English texts, and explore how translators can use the ecological translation theory to adapt to the complex translational ecological environment. The quality of foreign translations.
\end{abstract}

\section{Development of Ecological Translation Studies}

Eco-translatology is a new translation research theory emerging in translation research in recent years. For the first time, it introduced the concept of ecology into the study of translation studies, and realized the "ecological turn" of translation studies.

The study of translation studies provides a new perspective. The essence of ecological translation studies is a cross-disciplinary study between the two departments of Ecology and Translatology. "It focuses on the integrity of the translation ecosystem. From the perspective of ecological translation studies, it makes a new description and interpretation of the nature, process, standards, principles and methods of translation and translation phenomena in the narrative way of ecological translation."

Translation adaptation theory is the theoretical basis of ecological translation studies. Translation adaptation theory is based on Darwin's theory of "Adaptation Selection" in the theory of biological evolution, and its translation philosophy emphasizes "translator-centered". Eco-translatology believes that the process of translation is the process of the translator adapting and selecting the entire translational eco-environment. The so-called translational ecological environment refers to "the collection of the text, cultural context and "translation community" and "the combination of spirit and matter". [1] The translation of the ecological environment is crucial for the production of translated texts and is a factor that restricts the translator's adaptation and selection. In the practice of translation, the translation of the ecological environment can affect the content style of the text, and the small can affect the words and sentences. The basic theory of ecological translation studies simply summarizes the translation method as "three-dimensional transformation". That is, under the influence of the entire translation ecological environment, the translator should follow the principle of multi-dimensional adaptation and adaptive selection, from language dimension and cultural dimension. The three dimensions of communication dimension adapt the text to the best translation, in order to achieve the best translation, which is the highest translation of "integration adaptation choice" [2]. 


\section{The Status of Cultural Translation in English Web Pages}

Under the background of the accelerating globalization process, China's international cultural exchanges have become more frequent. Chinese universities need to strengthen their external publicity to attract scholars, teachers and students from all over the world to come to China for academic exchanges and learning. The English webpage of colleges and universities serves as an external publicity window, which is mainly used to publicize schools, attract foreign students, conduct foreign cooperation in running schools and international academic exchanges and cooperation. Therefore, the quality of English web pages in domestic universities plays an important role in the school's external publicity.

In the "China Knowledge Network", the author uses "College English Webpage" and "College English Website" as keywords, and only 16 related papers are covered, covering communication, rhetoric, teleology, cross-cultural, localization, and ecology. Equal perspective. Zhang Xinjun and Yang Hui analyzed the common problems in the English translation of Chinese college web pages in the "Investigation of English Translation of University Web Pages".[3] Fan Yong, Wen Jun, and Zhu Zijun used the principle of translation teleology as the theoretical framework, and analyzed the phenomenon of functional, cultural and linguistic translation errors in the English version of the "School Profile" of some key university websites in China. Qiu Chunan and Ren Xiaoyu studied the translation mode of college web pages from the perspective of communication. Lei Peihua analyzed the rhetorical persuasive nature of foreign propaganda translation, and selected typical cases from the university web pages to carry out Chinese and English propaganda rhetoric. Moreover, there is only one research from the perspective of "ecological translation studies". From the perspective of ecological translation studies, Jia Heping explores the translation of English from local colleges and universities in Henan Province as a research object, and discusses how to achieve pragmatic equivalence. Generally speaking, these research results are far from enough compared with their importance. There are still many shortcomings and limitations in the study of English translation of university websites in terms of breadth and depth, especially from the perspective of ecology. Large excavation space.

\section{Research on English Web Pages in Universities from the Perspective of Ecology}

Each culture is special, and each special culture is reflected in the language that carries the culture, thus forming a cultural characteristic word. English is currently the most widely used language in the world. Studying the translation of English cultural features plays a crucial role in the success of intercultural communication. Based on the understanding of ecological translation studies, the translation of English cultural characteristic words can not only stay in the literal translation. The translator should analyze the original text from multiple dimensions, improve their adaptability and initiative selection consciousness, and achieve translation and original text. Maintain a high degree of consistency in speech and content.

$\mathrm{Hu}$ Gengshen pointed out that the translation method of translation adaptation theory is to focus on the transformation between "three dimensions". [1] "Three-dimensional": Adaptation of language dimension, cultural dimension and communicative dimension. The translator needs to complete the adaptive choices at these three levels, so as to obtain the translation with the highest integration and adaptation, and realize the pragmatic equivalence in the true sense. The author selected the English translation materials of the 211 key construction schools in several countries of Hunan Province as specific corpus. The characteristics are: the international influence is relatively large, and there are higher requirements in the external publicity effect, which can fully reflect the English translation of Chinese college websites. The popularity problem that exists in it. The introduction part of the school is the window for the external dissemination of information. It is of great research value to analyze the adaptability of text language, structure and pragmatic effect from the perspective of "three-dimensional" which can be seen in figure 1 . 


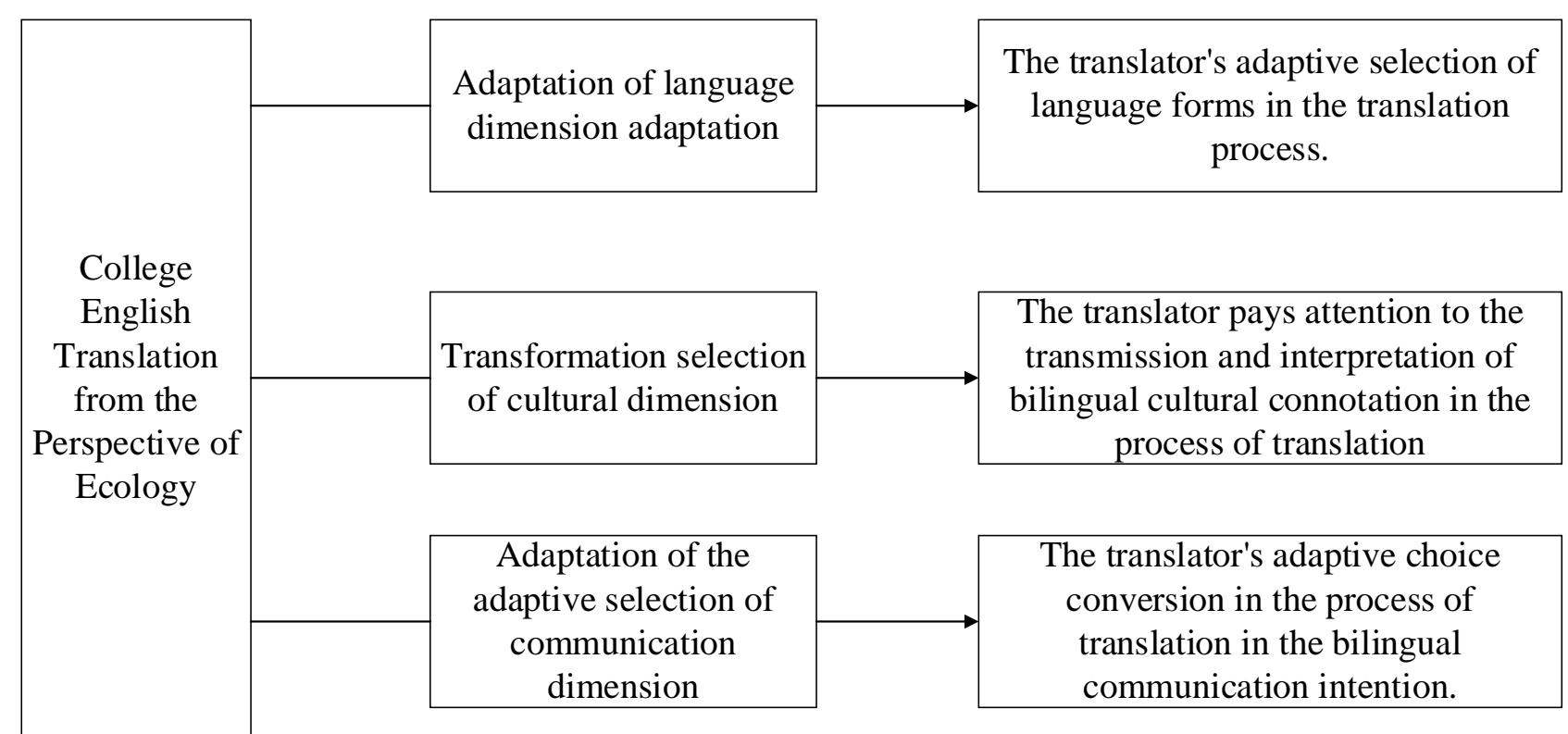

Figure 1. The format of translation adaptation theory

(1) Adaptation and selection in language dimension

Translation is an activity that transfers information content and changes language forms. Translator's "adaptive selection transformation of language dimension", that is, the translator's adaptive selection transformation of language forms in the translation process. The adaptive selection transformation of this language dimension is carried out in different aspects and at different levels. In the Chinese translation of English cultural characteristic words, in order to preserve the cultural characteristics of the original text and help the target readers to better understand the authentic English culture, transliteration or transliteration plus interpretation can be used to translate English cultural characteristics.

In English there is a large vocabulary which is characterized by a great number of synonyms. In representing the exact meaning and natural effect in translation, the translator should Choose the most appropriate word by making clear of the different connotations of the synonyms.

For example, at the time of translation, almost all universities in Shaanxi use the word "University", including a number of Vocational and private colleges that provide three - year and four - year education courses. However, in the American traditional dictionary, the university is defined as a higher education institution, which includes the teaching and research facilities of graduate and undergraduate colleges to grant more than a bachelor's degree. In this dictionary, the university is interpreted as a higher education institution, granting only a Bachelor of Arts and a Bachelor of Science degree. In the Macmillan English Chinese dictionary, a university usually means that a student over the age of 18 receives a training that does not usually have a degree. Students who receive a degree will receive a college degree. However, higher vocational colleges which do not provide bachelor's degree or above should not be named universities.

For instance 1, Shaanxi Fashion Engineering University should be translated into Shaanxi Fashion Engineering College.

(2) Adaptation and choice in cultural dimension

Culture and translation are inextricably linked. In 1980, the translation study by Susan Bassnett, a British scholar, pointed out that translation studies should focus on translation from the cultural level and open up translation studies. The door to the "cultural turn". In the process of translation, cultural factors are a problem that has to be considered. Since the original language culture and the translated language culture often have differences in nature and content, in order to avoid misinterpreting the original text from the perspective of the translated language culture, the translator not only needs to pay attention to the language conversion of the original language, but also needs to adapt to the entire cultural system to which the language belongs. And pay attention to the transmission of bilingual culture in the process of translation. [4] English cultural words carry a unique English culture. When the translation is true, the translator must respect this culture and 
retain this culture as much as possible. But at the same time, it should be noted that this kind of reservation does not only mean "alienation", but to make the reader understand the meaning and meaning of "different" as much as possible while being as "dissimilated"as possible.

For instance 2,among XJTU's outstanding alumni are Cai Wei, Huang Yanpei, Shao Lizi, Li Shutong, Ling Hongxun, Zou Taofen, Lu Dingyi, Hou Shaoqiu, Qian Xuesen,...

Xi'an Jiaotong University reviewed the history of the school and many alumni of different fields, which is a century-old school for Chinese readers. Talents come forth in large numbers, but they are famous for Western readers. They are meaningless to the meaning of the speaker and the status of the school. It is suggested that the characters should be introduced to the background or omitted.

(3) Communicative dimension

"Adaptation of the adaptive selection of communication dimension", that is, the translator's adaptive choice conversion in the process of translation in the bilingual communication intention. [4] The ultimate goal of the external propaganda is to achieve "intercultural communication". In addition to the "language dimension"and the adaptive choice of "cultural dimension", the adaptive choice of the communicative dimension in the external propaganda translation is particularly important. The translator must pay attention to the communicative intention in the original text, and the response of the target reader in the external translation directly tests whether the translation achieves true and effective communication. In the translation of foreign propaganda, the translation not only reflects the communicative intention of the original text, but also arouses the positive and effective response of the reader. In intercultural communication, the prevailing group or ethnocentrism, that is, the two sides of the communication will unconsciously use their own values and world views as the standard for explaining or judging everything. It is not uncommon for the translator to fail to take into account the cultural conflicts caused by the readers' beliefs and values. This is a typical "cultural translation mistake." [4] This led to the failure of intercultural communication. Most domestic universities are state-owned institutions, and the degree of government support and attention to colleges and universities is often the embodiment of school strength and status. Therefore, the external propaganda materials of domestic universities often emphasize the administrative subordination or level of their own schools, such as "national key universities" and "directives directly under the Ministry of Education". In contrast, the top universities in Western countries are mostly non-public universities, and whether the school has a public stereo system and the level and status of the school are not much related, such as "national key disciplines", "provincial and ministerial-level key scientific research institutions" and other information pairs. There is no positive significance for Western institutions with completely different values.

\section{Conclusion}

English translation plays an important role in understanding English culture and promoting intercultural communication. Don't make a hard copy in the process of translation, just pass the language information. It is necessary to proceed from the entire translation ecosystem and pay attention to the reader's feelings and acceptability. The difference between the source language and the target language requires the translator to make corresponding adaptations and choices according to the ecological environment of the translation, and dynamically adjust the translation strategy to obtain survival. The introduction of college web pages is an information-based text, focusing on objective and clear-cut vocabulary. The content is best based on the facts. Chinese text usually combines different types of information such as campus location, school history, teaching and research conditions into one as a translated language text, which is complicated and lengthy. The English text is clear, concise, and more focused on the transmission of objective facts and practical information. This paper studies the translation errors of the English translation of Chinese college web pages from the three dimensions of language dimension, culture dimension and communication dimension. The problems in the translation reflect the lack of attention of Chinese universities in web page translation and the lack of strict quality control. This is unfavorable to the external publicity of colleges and universities. 


\section{References}

[1]Hu Gengshen. Research focus and theoretical perspective of ecological translation studies [J]. Chinese translation,2011(2): 5+9.

[2]Hu Gengshen. Interpretation of Ecological Translation Studies [J] . Chinese Translation, 2008(6): $11+15$.

[3]Bai, Ruo Ran. "On the English Translation of Shaanxi Snacks from the Perspective of Skopos Theory." Overseas English, 2016(15):147+149.

[4]Yin, Xi Fang, and Y. E. Cong-Ling. "A Tentative Study on the Ecological Translation of LiQinzhao's Shengshengman from the Perspective of the Conversion of Subject and Object." Journal of University of South China.2011(10):483+490 